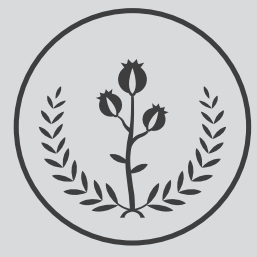

FUCS

\title{
Rep

\section{Cuidados de enfermería para la persona adulta, diabética con hipoglucemia: revisión integrativa}

\section{Nursing care for the adult diabetic experiencing hypoglycemia: an integrative review}

Ana Julia Carrillo

Karolain Meliza Beltrán ${ }^{\mathrm{b}}$

Diana Marcela Bolívar ${ }^{b}$

Sandra Milena Hernández ${ }^{\circ}$

Diana Cristina Henao MD $^{\mathrm{d}}$

a Facultad de Enfermería. Enfermera, Magister Administración en Salud, Grupo Perspectivas del Cuidado, Fundación Universitaria de Ciencias de la Salud. Bogotá DC, Colombia.

${ }^{b}$ Facultad de Enfermería, Fundación Universitaria de Ciencias de la Salud. Bogotá DC, Colombia.

${ }^{c}$ Enfermera. PhD en Ciencias de la Salud, Mg en cuidados de salud para la promoción de la autonomía de las personas y la atención a los procesos de fin de vida, Facultad de Enfermería, Grupo Perspectivas del Cuidado. Fundación Universitaria de Ciencias de la Salud. Bogotá DC, Colombia.

${ }^{d}$ Medicina Interna, Endocrinología, Hospital Universitario San Ignacio, Pontificia Universidad Javeriana, Bogotá DC, Colombia.

\section{R E S U M EN}

Introducción: La hipoglucemia es la complicación más frecuente del tratamiento con insulina en adultos. Los eventos de hipoglucemia severa se asocian con complicaciones a corto, mediano y largo plazo en pacientes con diabetes mellitus. Una de las estrategias para reducir la frecuencia de hipoglucemia son las intervenciones de enfermería y aunque hay pocos estudios que las enuncian de manera explícita, se ha publicado respecto a las necesidades de dichos pacientes, que permiten determinar diagnósticos de enfermería y a partir de ellos establecer metas e intervenciones para el cuidado de dichos pacientes. Objetivo: identificar los cuidados de enfermería para prevenir y controlar los eventos de hipoglucemia en pacientes adultos diagnosticados con diabetes mellitus. Metodología: revisión integrativa, se realizó en seis fases: planteamiento de la pregunta PICO; búsqueda en bases de datos y metabuscadores; lectura crítica; análisis, clasificación, validación por nivel de evidencia y grado de recomendación, y presentación de la información. Resultados: la revisión reportó cinco categorías: factores de riesgo y protectores, miedo a la hipoglucemia, atención brindada al paciente, disminución de la hipoglucemia y descripción

\section{INFORMACIÓN DEL ARTÍCULO}

Historia del articulo:

Fecha recibido: diciembre 6 de 2019 Fecha aceptado: agosto 5 de 2020
Autor para correspondencia.

Sra. Ana Julia Carrillo:

ajcarrillo@fucsalud.edu.co
DOI

10.31260/RepertMedCir.01217372.1001 
del impacto de la hipoglucemia en los pacientes. Conclusiones: a partir de las necesidades reportadas en las 5 categorías de los resultados se determinaron características definitorias y factores relacionados que permitieron formular diagnósticos de enfermería y determinar como principales intervenciones: enseñanza del proceso de enfermedad, medicamentos prescritos, entrenamiento de asertividad, manejo de la hipoglicemia, nutricional y de la medicación, mejorar el afrontamiento, enseñanza individual, facilitar el aprendizaje y potenciación de la disposición de aprendizaje.

Palabras clave: diabetes mellitus, hipoglucemia, atención de enfermería.

(C) 2021 Fundación Universitaria de Ciencias de la Salud - FUCS. Este es un artículo Open Access bajo la licencia CC BY-NC-ND (http:// creativecommons.org/licenses/by-nc-nd/4.0/)

\section{ABSTRACT}

Introduction: hypoglycemia is the most common complication of insulin therapy in adults. Events of severe hypoglycemia are associated with short, medium and long term complications in patients with diabetes mellitus (DM). One of the strategies to reduce the frequency of hypoglycemia are nursing interventions and although there are few studies that explicitly describe them, there are publications on the needs of such patients, allowing the determination of nursing diagnoses and based on them, setting goals and interventions to deliver care for these patients. Objective: to identify nursing care interventions to prevent and control hypoglycemic events in adult patients diagnosed with DM. Methodology: an integrative review was conducted in six phases: posing the PICOT question; search in databases and metasearch engines; critical reading; analysis, classification, validation by level of evidence and degree of recommendation, and data presentation. Results: the review reported five categories: risk and protective factors, fear of hypoglycemia, care provided to the patient, decrease in hypoglycemia and description of the impact of hypoglycemia on patients. Conclusions: Based on the needs reported in the resulting five categories, defining characteristics and related factors were determined allowing the formulation of nursing diagnoses and identifying the following as the main nursing interventions for hypoglycemia management: teaching of the disease process, prescribed medication, assertiveness training, hypoglycemia management, nutritional and medication therapy, improving coping, individual teaching, facilitating learning and empowering the willingness to learn.

Key words: diabetes mellitus, hypoglycemia, nursing care.

(C) 2021 Fundación Universitaria de Ciencias de la Salud - FUCS. This is an open access article under the CC BY-NC-ND license (http://creativecommons.org/licenses/by-nc-nd/4.0/).

\section{N T ROD U C C I Ó N}

La diabetes mellitus (DM) es una enfermedad metabólica crónica que se caracteriza por hiperglucemia; lo anterior desencadenado por diversas etiologías las cuales conllevan a que los niveles de glucosa en sangre sean elevados. ${ }^{1}$ Se han identificado cuatro tipos de DM. La DM tipo 1 o también llamada insulinodependiente, juvenil o de inicio en la infancia, caracterizada por una producción deficiente de insulina y requiere suministro exógeno diario; la DM tipo 2 se presenta en la edad adulta, por la incapacidad del organismo para hacer uso eficaz de la insulina; la diabetes gestacional, y los tipos específicos de diabetes debido a diferentes causas no relacionadas con las anteriormente descritas. ${ }^{2,3}$

El último reporte del Atlas de la Federación Internacional de Diabetes muestra que aumentará el número personas que viven con diabetes de 417 millones para el año 2030 a 486 millones para el 2045, reflejando un incremento considerable en la población, lo que implicará desafíos económicos y de salud pública inevitables. ${ }^{4}$ Colombia se posicionó en el segundo lugar de los cinco países de Suramérica con mayor prevalencia de diabetes, con 2.781.082 casos, que excedió las cifras presentadas por países como Argentina, Chile y Venezuela. ${ }^{5}$ El Ministerio de Salud y Protección Social (Minsalud) en el Análisis de Situación en Salud (ASIS) 2017 encontró que Bogotá tiene una prevalencia de $20.20 \%{ }^{6}$ Las cifras reportadas, muestran la necesidad de abordar y profundizar sobre las secuelas de la diabetes que son perjudiciales y ponen en peligro la vida y la calidad de la misma; es preciso mencionar que cuando la diabetes no se atiende de manera eficiente se presentan complicaciones como cetoacidosis diabética (CAD), coma hiperosmolar, alteraciones cardiacas, vasculares, lesiones oculares (retinopatía diabética, ceguera), renales (insuficiencia renal) y en los nervios (neuropatía), por otro lado aumenta la incidencia de accidentes cerebrovasculares. La disminución del flujo sanguíneo junto con la neuropatía a nivel periférico causa en los pacientes alto riesgo de sufrir úlceras e infecciones especialmente en miembros inferiores que 
pueden llevar incluso a la amputación, frente a la diabetes gestacional se evidencia incremento en anomalías congénitas, muerte perinatal, mortalidad materna, preeclampsia, eclampsia, macrosomía fetal y distocia de hombro en el niño. ${ }^{7}$ Para atenuar la incidencia, prevalencia y presencia de complicaciones en pacientes diagnosticados con DM se han planteado diferentes estrategias a nivel internacional y nacional; uno de ellas es el Marco Mundial de Vigilancia Integral que incluye nueve metas, donde se destacan la detección del aumento de la diabetes y la obesidad, además del tratamiento farmacológico y asesoramiento (incluido el control de la glucemia) de al menos un $50 \%$ de las personas que lo necesitan; para cumplir se proporcionó una hoja de ruta y opciones de normativa. Así mismo se cuenta con el plan de acción mundial para la prevención y el control de las enfermedades no transmisibles (ENT) 2013-2020 de la OMS; en 2015 en la Asamblea General de las Naciones Unidas se adoptó la agenda 2030 para el desarrollo sostenible que plantea como objetivo reducir en un tercio la mortalidad prematura atribuible a las ENT, lograr la cobertura sanitaria universal, y proporcionar acceso a los medicamentos esenciales a un costo asequible para reducir la mortalidad evitable atribuible a la diabetes. ${ }^{7}$

En Colombia existen las Rutas Integrales de Atención en Salud (RIAS) y Guías de Práctica Clínica para la diabetes, incluidas en el Plan Decenal de Salud Pública (PDSP) 20122021, 8 adicionalmente Minsalud promueve la estrategia 4x4 que consiste en adoptar cuatro hábitos saludables (actividad física, evitar el consumo de tabaco y de alcohol, promocionar la alimentación saludable, rica en frutas y verduras) para disminuir la aparición de enfermedades cardiocerebro-vasculares, la diabetes, la enfermedad pulmonar obstructiva crónica y el cáncer. ${ }^{9}$ Sin embargo, en pacientes diagnosticados con DM, prevalece la aparición de eventos de hipoglucemia caracterizados por síntomas como por temblor, taquicardia, diaforesis, mareo, hambre, cansancio, ansiedad, visión borrosa, cefalea e irritabilidad; ${ }^{10}$ que tiene como origen un inadecuado cuidado de la enfermedad ya sea porque la persona deja de consumir una de las comidas, hace más ejercicio del habitual o cuando la dosis del hipoglucemiante es demasiado alta; llegando a ocasionar hipoglucemias severas con convulsiones y pérdida del conocimiento. ${ }^{7}$

LaSociedad Española de Endocrinología y Nutrición, estima que de 2 a $4 \%$ de las muertes de pacientes diagnosticados con DM tipo 1 se atribuyen a hipoglucemias. ${ }^{11}$ Adicional a esto un estudio de tipo observacional desarrollado en el Reino Unido, mostró que los pacientes con DM tipo 2 presentan un riesgo de $7 \%$ de sufrir hipoglucemia severa en los primeros años de evolución. ${ }^{12}$ La hipoglucemia se clasifica en niveles, según la guía ADA 2019: el nivel 1 o valor alerta cuando la glicemia es $<70 \mathrm{mg} / \mathrm{dL}$ (3.0 mmol/L) requiere ajustes de dosis de hipoglucemiantes, se necesitará tratamiento con carbohidratos de acción rápida; el segundo nivel o hipoglucemia clínicamente significativa $<54 \mathrm{mg} /$
dL (3.0 mmol/L); el tercer nivel o hipoglucemia severa no tiene un umbral de glucosa específico, se caracteriza por la alteración cognitiva y/o requerimiento de ayuda de terceros para resolver el episodio. ${ }^{13,14}$

Con base en la anterior información y la revisión de hipoglucemia en pacientes diabéticos desarrollado en Montevideo, se concluye que la hipoglucemia al no ser de fácil registro por la falta de control y en algunos casos por la poca o nula conciencia de la sintomatología manifestada por el paciente, se encuentra subdiagnosticada y por consiguiente no había generado el interés de realizar estudios investigativos que aborden el cuidado integral del paciente que presenta episodios de hipoglucemia a lo largo de su enfermedad y teniendo en cuenta que es una emergencia que requiere ser diagnosticada y tratada rápidamente para evitar las complicaciones desencadenadas por la misma. ${ }^{15}$ Adicionalmente, consciente de las consecuencias del manejo inadecuado de esta enfermedad, el grupo investigador se plantea como objetivo conocer los cuidados de enfermería para la atención del paciente adulto con diabetes mellitus con eventos de hipoglucemia.

\section{METODOLOGÍA}

Se realizó una revisión integrativa de la literatura, porque permite la incorporación de evidencias a la práctica clínica mediante la recolección, análisis y síntesis de los resultados de múltiples estudios publicados, que contribuyen a la profundización del conocimiento del tema de investigación y dan soporte a la toma de decisiones. ${ }^{16}$ Este estudio se desarrolló en seis fases con base en la propuesta de Mendes, Campos y Galvao. ${ }^{16}$

En la primera fase se estableció la siguiente pregunta orientadora: ¿Cuáles son los cuidados de enfermería para prevenir y controlar los eventos de hipoglucemia en el paciente adulto con diabetes mellitus? En la segunda fase se realizó una búsqueda en las bases de datos CINAHL, LILACS, OVID NURSING, COCHRANE y en el metabuscador PUBMED. Se utilizaron las ecuaciones de búsqueda: Diabetes mellitus AND hipoglucemia AND cuidados enfermería, Diabetes Mellitus AND hypoglycemia AND Nursing care y Diabetes mellitus AND Hipoglucemia AND Cuidados de Enfermagem construidas a partir de los términos MeSH y DeCs. Se establecieron como criterios de selección: investigaciones cuyos sujetos son personas mayores de 18 años diagnosticados con diabetes mellitus tipo I y II, publicados o actualizados entre 2014 y el primer semestre de 2019, en los idiomas inglés, portugués y español. Se incluyeron estudios observacionales, experimentales, cualitativos y de investigación secundaria para proporcionar una comprensión más completa del tema de interés. Se excluyeron estudios de población diagnosticada con diabetes gestacional y otros tipos de diabetes derivadas de efectos secundarios medicamentosos o por procesos oncológicos. En la tercera fase se realizaron análisis y síntesis 


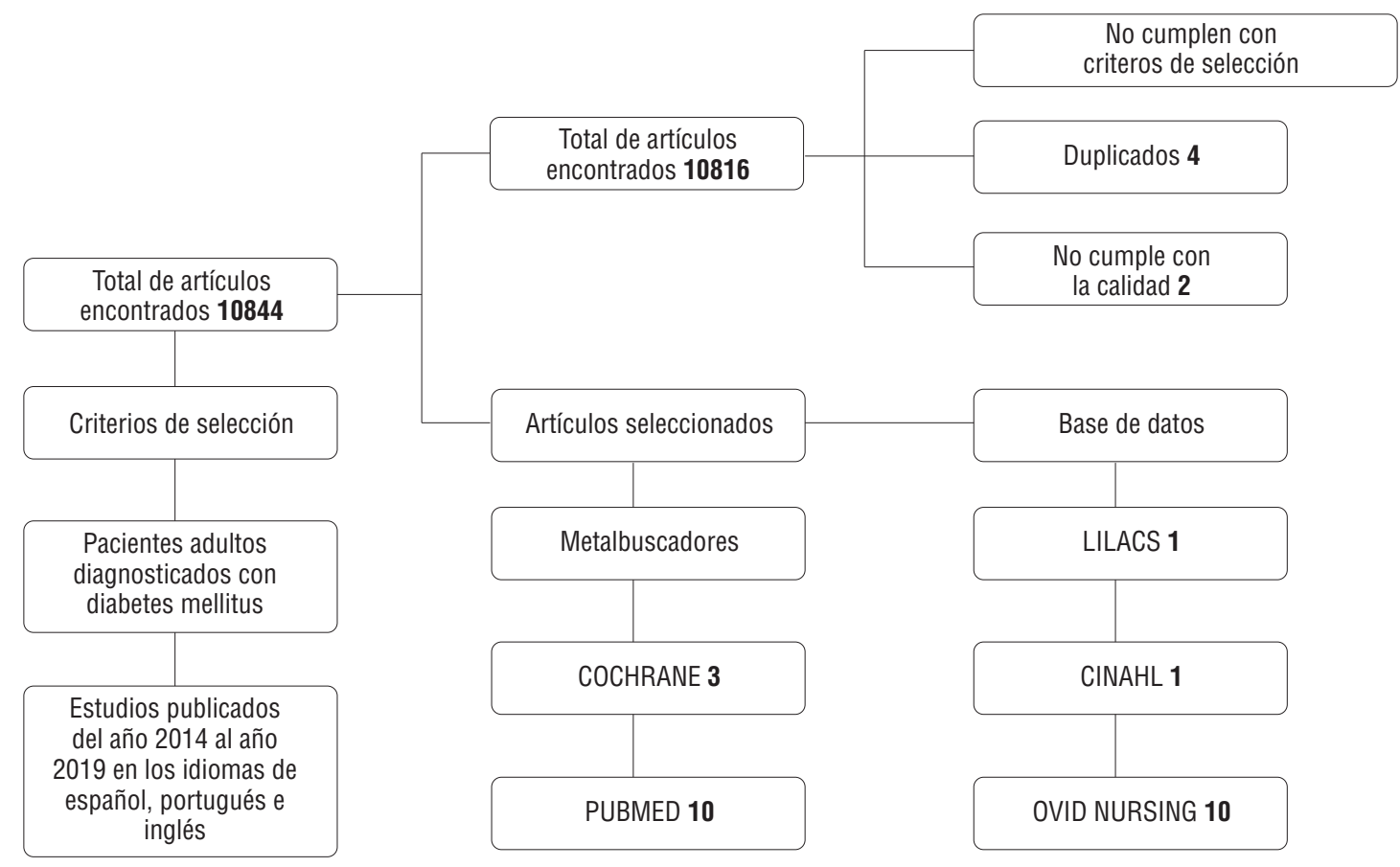

Figura 1. Flujograma de búsqueda. Fuente: las autoras.

de la información extraída de cada estudio plasmándola en una base de datos elaborada en Office Microsoft Excel ${ }^{\circledR}$. La búsqueda inicial reportó un total de 10.844 artículos; después de aplicar los criterios de selección, exclusión y las listas de chequeo de calidad metodológica se seleccionaron 25 investigaciones, que contenían información relevante para dar respuesta a la pregunta de investigación (figura 1).

En la cuarta fase se realizó una revisión por pares, para ello se aplicaron los formatos de lectura crítica CONSORT para los Ensayos Clínicos Aleatorizados, TREND en estudios cuasiexperimentales, STROBE para estudios observacionales, AMSTAR para revisiones sistemáticas y el formato CASPE en los estudios de tipo cualitativo; para el cumplimiento de calidad metodológica se determinó como nivel mínimo de evaluación 17, 16, 13, 16 puntos y 3 ítems respectivamente. ${ }^{17}$ Las listas de chequeo evalúan la validez interna y externa de los estudios de acuerdo al diseño de la investigación. Posteriormente se clasificaron los resultados por nivel de evidencia y grado de recomendación, utilizando la propuesta del Centre for Evidence-Based Medicine (CEBM) de Oxford. ${ }^{18}$ En la quinta fase se realizó una síntesis de los resultados en 5 categorías temáticas: factores de riesgo y protectores, miedo a la hipoglucemia, atención brindada al paciente, disminución de la hipoglucemia, y el impacto de la hipoglucemia. En la última fase se interrelacionaron los resultados de la revisión con los factores relacionados y características definitorias para formular los Diagnósticos de Enfermería definidos por la North American Nursing Diagnosis Association (NANDA), sus respectivas metas de acuerdo con la Nursing Outcomes Classification (NOC), las intervenciones establecidas por la Nursing interventions classification (NIC) y las actividades descritas para cada una de ellas. Se tuvieron en cuenta las consideraciones éticas establecidas por el Ministerio de Salud en Colombia en la resolución 8430 de 1993 que clasifica este estudio como una investigación sin riesgo y en la ley 44 de 1993 que regula los derechos de autor los cuales se garantizan con la citación. ${ }^{19,20}$

\section{RES U LTA D OS}

De acuerdo con los criterios de búsqueda se seleccionaron 28 artículos, de los cuales 25 cumplieron los criterios establecidos para la lectura crítica, 4\% en español y 96\% en inglés; la categorización por año de publicación reportó que $11 \%$ son de 2014, 22\% de 2015, $11 \%$ de 2016, 44\% de 2017 y $12 \%$ de 2018 . Referente al tipo de estudio $4 \%$ corresponde a metaanálisis, $8 \%$ a revisiones de la literatura, 16\% ensayos clínicos aleatorizados, $4 \%$ estudios mixtos, 28\% descriptivos de corte transversal, $8 \%$ cualitativos, $12 \%$ casos y controles, $16 \%$ cohortes y $4 \%$ cuasiexperimentales. Según el país de publicación Estados Unidos se encuentra en el primer lugar con $24 \%$, seguido de Dinamarca y Noruega 12\%; China y Escocia 8\%; Canadá, Italia, Suecia, Japón, Perú, Brasil, Alemania, Reino Unido y Holanda 4\% cada uno. Respecto al nivel de evidencia y grado de recomendación, $62.5 \%$ tiene recomendación favorable, 29\% recomendación extremadamente favorable y $8.5 \%$ ni se recomienda ni se desaprueba. Los artículos previamente seleccionados después de la extracción y análisis de la información para identificar cuidados de enfermería 
para la prevención y control de la hipoglucemia, reportaron 5 categorías (tabla 1). En la primera categoría se incluyeron los factores de riesgo y protectores asociados a eventos de hipoglucemia, que incrementan su aparición y dependen del tratamiento brindado al paciente; la inasistencia de los miembros de la familia y el inadecuado autocuidado del paciente entre otros. Por otro lado, se encontraron factores protectores como el tratamiento de insulina subcutánea y la medición diaria de los niveles de glucosa en sangre. ${ }^{21-26} \mathrm{La}$ categoría de miedo a la hipoglucemia reporta que se ve afectada la conciencia de los pacientes referente a los síntomas de hipoglucemia, creando barreras que impiden el control de la misma, lo que a su vez se comporta como un factor de estrés psicológico que puede aumentar el nivel de consumo de calorías y disminuir el ejercicio físico. ${ }^{27-29} \mathrm{En}$ la categoría atención brindada al paciente la evidencia muestra que las personas diabéticas requieren un equipo de salud capacitado, que los ayude a generar control general de la enfermedad para evitar complicaciones como la hipoglucemia, mediante una atención personalizada, se recomienda atención por la especialidad de endocrinología, que permita el análisis efectivo de la situación clínica actual para establecer las metas terapéuticas deseadas con un régimen de medicamento adecuado. ${ }^{30-34}$ En la categoría disminución de la hipoglucemia se evidencia en los estudios la implementación de intervenciones y planes de acción encaminados a la atención y educación del paciente con el objetivo de reducir la hipoglucemia en el paciente adulto con DM. ${ }^{35-40}$ La categoría impacto de la hipoglucemia muestra que gran proporción de los pacientes presentan hipoglucemias severas, requiriendo así la asistencia de una ambulancia médica; frente a la hipoglucemia nocturna se evidenció que afecta potencialmente la capacidad del paciente para despertarse y responder con una ingesta adecuada de carbohidratos, entre otros síntomas. ${ }^{41-45}$

Tabla 1. Categorías temáticas

\section{Factores de riesgo y protectores}

Wohland $\mathrm{T}$, Holstein $\mathrm{T}$, Patzer 0, Mende M, Tiemann T, Koch-Tessarek $C$, et al. New risk and protective factors for severe hypoglycaemia in people with type 1 diabetes. 2017 may; 27(5): [Page 407-414]

Sonoda N, Morimoto A, Ugi S, Morino K, Sekine 0, Nemoto K, et al. Predictors for Mild and Severe Hypoglycemia in Insulin-Treated Japanese Diabetic Patients. 2015 june; 10(6).

Moutairou A, Roussel R, Charbonnel B, Leye A, Detournay B, Mohammedi K, Potier L. Predicting severe hypoglycaemia with self-monitoring of blood glucose in type 1 diabetes. 2017 september; 43(4): [Pages 392-394].

Rooijackers H, Wiegers E, Graaf M, Thijssen D, Kessels R, Bastiaan $T$ et al. A Single Bout of High-Intensity Interval Training Reduces Awareness of Subsequent Hypoglycemia in Patients With Type 1 Diabetes.2017 july; 66(7): [Pages 1990-1998].
NR: 2B

GR: $B$

Recomendación favorable. ESCALA STROBE

17 puntos

NE: 2B

GR: $B$

Recomendación favorable ESCALA STROBE

15 puntos

NE: 2B

GR: B

Recomendación favorable

ESCALA STROBE

16 puntos

NE: 1B

GR: A

Recomendación

extremadamente favorable

ESCALA CONSORT

22 puntos
El artículo concluye que los factores de riesgo asociados a hipoglucemia son: la falta de atención de enfermería (OR 4.88), tratamiento con insulina NPH (Neutral Protamine Hagedorn) (OR 3.68), y la falta de conciencia de hipoglucemia (OR $2,06)$. Además, el $50 \%$ de los episodios se asociaron con 31 pacientes que presentaron eventos previos.

Por otro lado se encontraron factores que reducen ese riesgo cómo el tratamiento con CSII (infusión continua de insulina subcutánea), pacientes con depresión y tratamiento con insulinas de acción corta.(21)

Este estudio reporta que la falta de asistencia de los miembros de la familia en el momento de la inyección de insulina y de recibir un tratamiento intensivo con insulina fueron factores predictivos de hipoglucemia grave. Además, la falta de asistencia de los miembros de la familia en el momento de la inyección de insulina y el consumo actual de alcohol fueron factores predictivos de hipoglucemia leve. (22)

Los resultados de este estudio permiten inferir que la hipoglucemia leve es un factor predictivo importante de los episodios de hipoglucemia grave en pacientes con Diabetes Tipo 1. (23)

La evidencia muestra que una breve serie de ejercicios de intervalo de alta intensidad suprime los síntomas de hipoglucemia posterior en pacientes con diabetes tipo 1, pero no afecta la conciencia en pacientes con conciencia deficiente de hipoglucemia IAH (conciencia de hipoglucemia alterada). El papel del lactato inducido por el ejercicio en la mediación de los efectos supresores de HIIT (Entrenamiento por intervalos de alta intensidad) en la conciencia de hipoglucemia debe explorarse más a fondo. La reducción de las respuestas sintomáticas puede aumentar el riesgo de hipoglucemia después del ejercicio intensivo. (24) 


\begin{tabular}{l}
\hline \\
Rosenstock J, Fonseca V, \\
Schinzel S, Dain M, \\
Mullins P, Riddle M. \\
Reduced risk of hypogly- \\
cemia with once-daily \\
glargine versus twice-dai- \\
ly NPH and number \\
needed to harm with NPH \\
to demonstrate the risk of \\
one additional hypoglyce- \\
mic event in type 2 \\
diabetes: Evidence from a \\
long-term controlled trial. \\
2014 september - \\
october]; 28(5): [Pages \\
742-749]. \\
Almanza 0, Chia E, De la Cruz \\
A, Tello T, Ortiz P. Frecuencia \\
de factores asociados a \\
hipoglucemia en el adulto \\
mayor diabético admitido \\
en el servicio de emergen- \\
cia de un hospital \\
nacional. 2017 junio; 28(2): \\
[Páginas 93-100].
\end{tabular}

\section{Factores de riesgo y protectores}

NE: $1 B$

GR: A

Recomendación

extremadamente favorable

ESCALA CONSORT

23 puntos

NE: $2 B$

GR: $B$

Recomendación favorable

ESCALA STROBE

17 puntos
El artículo concluye que, si bien las dosis totales diarias de insulina fueron similares con glargina y NPH, hubo aproximadamente un $29 \%$ menos de eventos hipoglucemiantes informados con glargina en comparación con el tratamiento con NPH. En consecuencia, se podría argumentar que la mayor frecuencia de hipoglucemia con NPH puede estar relacionada con la Hemoglobina Glicosilada (HbA1c) ligeramente más baja. (25)
El artículo reporta como factores de riesgo de hipoglucemia: una inadecuada alimentación, los fármacos hipoglucemiantes y sus dosis, la presencia de comorbilidades, especialmente Insuficiencia renal, síndromes geriátricos, depresión y existencia de dependencia funcional. (26)

\section{Hatle H, Bjørgaas M, Ro T, Olsen S, Đsvold B. Fear of hypoglycaemia and its relation to hypoglycaemia awareness and symptom intensity in Type 1 diabetes. 2018 march; 137: [Pages 213-220]. \\ Martyn-Nemeth P, Quinn L, Penckofer S, Park C, Hofer V, Burke L. Fear of hypoglycemia: Influence on glycemic variability and self-management behavior in young adults with type 1 diabetes. 2017 april; 31(4): [Pages 735-741] \\ Martyn P, Schwarz F, Mihailescu D, Nemeth J, Quinn L. Fear of hypogly- cemia in adults with type 1 diabetes: impact of therapeutic advances and strategies for prevention - a review. 2016 january-february; 30(1): [Pages 167-177].}

\section{Miedo a la Hipoglucemia}

NR: 3B
GR: B
Recomendación favorable
ESCALA STROBE
19 puntos
NE: 3B
GR: B
Recomendación favorable
ESCALA STROBE
14 puntos

NE: 3B
GR: B
Recomendación favorable
ESCALA STROBE
14 puntos

El estudio obtuvo como resultado que el miedo a la hipoglucemia es mayor en la población con hipoglucemia asintomática, antecedente de hipoglucemia severa y puntajes altos en la escala de hipoglucemia de Edimburgo, la cual, se utiliza para medir la presencia e intensidad de los síntomas de hipoglucemia.(27)

Las conclusiones del artículo refieren el miedo a la hipoglucemia crea barreras que impiden el control de glucemia, causando así un aumento en la variabilidad glucémica, además de afectar la conciencia de hipoglucemia por la ansiedad. Por otro lado al ser un factor de estrés psicológico en los pacientes hace que los comportamientos cambien causando incremento en el consumo de calorías y disminución de la actividad física realizada.(28)

El estudio concluye que a pesar de los refinamientos en la terapia con insulina y los avances en la tecnología de detección de glucosa en las últimas décadas, el miedo a la hipoglucemia (FOH) sigue siendo un factor disuasivo fundamental para el autocontrol de la diabetes, el bienestar psicológico y la calidad de vida (QOL) entre las personas con diabetes tipo 1.(29)

\section{Atención brindada al paciente}

Halperin I, Mukerji G, Maione M, Segal P, Wolfs M, Goguen $J$, etal. Adult Patient Perspectives on Care for Type 1 and Type 2 Diabetes Across the Institute of Medicine's 6 Domains of Quality. 2018 february; 42(1): [Pages 36-43]
NE: 5

GR: D

Ni se recomienda

ni se desaprueba.

\section{ESCALA CASPe}

Cumple con los tres primeros criterios
Las conclusiones de este artículo plantean el deseo de los pacientes por contar con un equipo informado, atento, disponible y comunicativo que ayude con el autocontrol y el control eficaz, centrado en el paciente y oportuno de la diabetes. Además, mostró que los pacientes deseaban evitar las complicaciones de la diabetes, incluida la hipoglucemia, los beneficios de compartir la planificación de la atención y lograr metas personalizadas. La atención eficiente y equitativa no se priorizó. (30) 


\section{Atención brindada al paciente}

Hao S, Zhang N, Folta A,
Yuan X,Liu L, Fan Li, et al.
Inpatient Glycemic
Management in Internal
Medicine: An Observatio-
nal Multicenter Study in
Nanjing, China. 2017
june; 33(8).
International Hypoglycae-
mia Study Group. Minimi-
zing Hypoglycemia in
Diabetes. 2015 august];
38(8):
1583-1591].
[Pages
Andreassen L, Sandberg
S, Kristensen G, Solvik U,
Skeide R. Nursing home
patients with diabetes:
Prevalence, drug
treatment and glycemic
control.2014 july; 105(1):
[Pages 102-109].

NE: 2B

GR: B

Recomendación favorable ESCALA STROBE

16 puntos

NE: 2B

GR: $B$

Recomendación favorable

\section{ESCALA STROBE}

15 puntos

NE: 2B

GR: B

Recomendación favorable

ESCALA STROBE

19 puntos
El estudio mostró que existe un control glucémico deficiente sobre todo en los pacientes diabéticos tratados en los departamentos diferentes a endocrinología, porque el 7,8\% tenían hipoglucemia.(31)

Las conclusiones de los artículos coinciden en que se debe tener especial cuidado al elegir la terapia hipoglucemiante para pacientes con deterioro cognitivo. El alto número de pacientes tratados con insulina, junto con varios registros de concentraciones bajas de glucosa en sangre y valores bajos de $\mathrm{HbA1c}$ sugieren que algunos pacientes están sujetos a un tratamiento excesivo. Las nuevas pautas recomiendan límites de $\mathrm{HbA} 1 \mathrm{c}$ menos estrictos para pacientes mayores y en consecuencia los hogares de ancianos noruegos deben ajustar sus objetivos de tratamiento. También debe aplicarse la planificación de la atención individual, especialmente en pacientes con alta variabilidad glucémica.(32-33)

\section{Atención brindada al paciente}

Jones G, Khan J, NR: 1C

Sainsbury C. Is all hypoglycaemia treated as equal? An observational study of how the type of diabetes and treatment prescribed priorto admission influences quality of treatment of inpatient hypoglycaemia. 2017 march; 54(3): [Pages 247-250].

\section{GR: A}

Recomendación favorable

\section{ESCALA STROBE}

15 puntos
Este estudio sugiere que la calidad de la atención de la hipoglucemia varía según el diagnóstico y la medicación. Tomando mayor relevancia los pacientes diagnosticados con DM tipo 1, sin embargo, en el estudio se hace referencia a que los pacientes con DM tipo 2 deben tener una vigilancia más estrecha de sus niveles de glucosa en sangre, debido a que los riesgos asociados con la hipoglucemia pueden ser mayores. (34)

\section{Disminución de la hipoglucemia}

Marelli G, Avanzini F, Lacuitti G, Planca E, Frigerio I, Busi G, et al. Effectiveness of a Nurse-Managed Protocol to Prevent Hypoglycemia Hospitalized Patients with Diabetes.2015 march.

Walfridsson A, Sehlberg M, Gillespie U, Dahlkvist J, Johansson $\mathrm{H}$. Diabetes treatment and hypoglycaemic episodes in elderly patients at nursing homes in Uppsala County.2016 june; 121(3): [Pages 179-183].

\section{NR: $1 C$}

GR: A

Recomendación extremadamente favorable ESCALA STROBE 18 puntos

NE: 2B

GR: $B$ Recomendación favorable. ESCALA STROBE 15 puntos
El estudio concluyó que las medidas tomadas por una enfermera redujeron significativamente la hipoglucemia en pacientes diabéticos hospitalizados tratados con insulina. Teniendo en cuenta que los eventos de hipoglucemia son frecuentes en el entorno hospitalario, se necesita garantizar que los pacientes tengan glucosa infundida durante un ayuno prolongado, consuman la cantidad planificada de carbohidratos con la dieta y, en caso de falta de apetito o consumo reducido de carbohidratos planificados, se administra una dosis más baja de insulina prandial al final de la comida. Esto debería convertirse en una estrategia preventiva de rutina en el hospital

Los resultados de este estudio sugieren que los pacientes ancianos con multimorbilidad y reporte de $\mathrm{HbA} 1 \mathrm{c}$ baja, que sufren episodios de hipoglucemia podrían beneficiarse de una reevaluación de sus tratamientos farmacológicos, incluidos los cambios de los agentes utilizados, la reducción de la dosis 0 incluso la suspensión de los tratamientos seleccionados. La HbA1c debe controlarse con frecuencia para garantizar que se mantenga dentro de niveles razonables.(36) 


\section{Disminución de la hipoglucemia}

Duncan D, Fitzpatrick D. Improving self-referral for diabetes care following hypoglycaemic emergencies: a feasibility study with linked patient data a $\mathrm{n}$ a I y s i s. 2016 february9]; 16(13).

Yu-xue C, Zheng-ren L, Ying $Y$, En-sheng $Y$, Xing-hua L. Effect of Recurrent Severe Hypoglycemia on Cognitive Performance in Adult Patients with Diabetes: A Meta-analysis.2017 october; 37(5): [Pages 642-648].

Vloemans A, Beers J, Wit M, Cleijne W, Rondags $S$, Geelhoed-Duijvestijn P. Et al. Research: Educational and Psychological Aspects Keeping safe. Continuous glucose monitoring (CGM) in persons with Type 1 diabetes and impaired awareness of hypoglycaemia: a qualitative study. 2017 july; 34(10): [Pages 1470-1476].

Pacheco A, Sande-Lee $\mathrm{S}$, Cássia R, Batista S, Marques J.Effects of a structured education program on glycemic control in type 1 diabetes.2017 december; 61(6): [Pages 534-541].
NE: $2 B$

GR: $B$

Recomendación favorable

ESCALA STROBE

17 puntos

GR: A

NE: $1 A$

Recomendación

extremadamente favorable

ESCALA AMSTAR

11 puntos

NE: 5

GR: D

Ni se recomienda ni se

desaprueba

ESCALA CASPe

Cumple con las tres

primeras condiciones.
El seguimiento telefónico proactivo por (NHS24) Scotland's national telehealth and telecare organisation fue factible y se percibió como útil para los pacientes; cuando la intervención se pudo implementar, fue altamente aceptable y considerada muy útil por la mayoría de los pacientes y médicos de ambulancia.(37)

El estudio concluye que los pacientes con DM deben conocer los perfiles de acción de la insulina utilizada y los efectos del ejercicio sobre la sensibilidad a la insulina. Es importante capacitar a los pacientes para que reconozcan los signos de alerta temprana y patrones de ocurrencia de hipoglucemia. El manejo clínico en pacientes adultos con DM debe incluir exámenes cognitivos para identificar pacientes que podrían beneficiarse de estrategias que reducen el riesgo de hipoglucemia severa.(38)

El estudio mostró que en la mayoría de los adultos con diabetes tipo 1 con riesgo hipoglucemia recurrente por hipoglucemia inadvertida, el uso de monitoreo continuo de glucosa (MCG) aumenta la sensación de control y seguridad. El MCG predice la tendencia de los niveles de glucosa y reduce eventos de hipoglucemia. Como resultado, disminuye la variabilidad glucémica y la hipoglucemia nocturna. Los participantes sintieron que su diabetes era más "estable" o "constante", sabiendo que obtendrían una alerta antes de que la glucosa en la sangre cayera demasiado baja lo cual
NR: 28

GR: $B$

Recomendación favorable

ESCALA STROBE

17 puntos
Los resultados de este estudio muestran que las personas con DMT1 tienen un déficit de información y/o conocimiento sobre esta condición, que, junto con la falta continua de apoyo profesional para el autocuidado, les impide lograr una autogestión efectiva. Por tanto, observaron mejoras significativas en el conocimiento y control glucémico después de las actividades del Programa de educación estructurada (SEP).(40)

\section{Impacto de la hipoglucemia}

Duncan E, Fitzpatrick D,lkegwuonu T, Evans J, Maxwell M. Role and prevalence of impaired awareness of hypoglycaemia in ambulance service attendances to people who have had a severe hypoglycaemic emergency: a mixed-methods study. 2018; $8(4)$.

\section{NE: 5}

GR: D

Ni se recomienda ni se desaprueba.

ESCALA STROBE

18 puntos

ESCALA CASPe

Cumple con los

tres primeros criterios
El estudio mostró que la prevalencia de hipoglucemia asintomática entre las personas que requieren una ambulancia después de un evento de hipoglucemia severa es más del doble comparado con la población general de personas con diabetes. Esto puede deberse a que las experiencias de las personas con hipoglucemia asintomática que requieren una ambulancia después de un evento hipoglucemia severa difieren de las personas con percepción normal de síntomas de hipoglucemia. (41) 


\section{Impacto de la hipoglucemia}

\begin{abstract}
Moheet A, Mangia B, Kumar A, Tesfaye A, Eberly C, Bai C, et al. Naltrexone for treatment of impaired awareness of hypoglycemia in type 1 diabetes: A randomized clinical trial. 2015 november-december; 29(8):

[Pages

1277-1282]
\end{abstract}

Jennum $\mathrm{P}$, Stender K, Rabøl R, Rye N, Chu P, Madsbad Sten. The Impact of Nocturnal Hypoglycemia on Sleep in Subjects With Type 2 Diabetes. 2015 november; 38(11): [Pages 2151-2157].

Gubitosi-Klug R, Braffett B, White N, Sherwin R, Service J, Lachin J. Risk of Severe Hypoglycemia in Type 1 Diabetes Over 30 Years of Follow-up in the DCCT/EDIC Study. 2017 august; 40(8): [Pages 1010-1016].

Christensen T, Cichosz S, Tarnow L, Randlov J, Kristensen L, Struijk J, et al. Hypoglycaemia and QT interval prolongation in type 1 diabetes bridging the gap between clamp studies and spontaneous episodes. 2014 september - october; 28(5): [Pages 723-728].
NE: $1 B$

GR: A

Recomendación extremadamente favorable ESCALA CONSORT

26 puntos

NE: $1 B$

GR: A

Recomendación extremadamente favorable ESCALA CONSORT 23 puntos

NE: $2 B$

GR: $B$

Recomendación favorable ESCALA STROBE

19 puntos

NE: 1B

GR: A

Recomendación extremadamente favorable

ESCALA TREND

16 puntos a investigación concluyó, que el tratamiento a corto plazo con naltrexona no mejoró el reconocimiento de los síntomas de hipoglucemia o la respuesta hormonal contrarreguladora durante la hipoglucemia inducida en sujetos con DMT1 e IAH.(42)

La evidencia mostró que la hipoglucemia nocturna afecta potencialmente la capacidad del paciente para despertarse y responder con una ingesta adecuada de carbohidratos. El estudio observó en pacientes con diabetes tipo 2 una reducción en la respuesta al despertar después de la hipoglucemia, aunque el efecto no fue inmediato (se produjo varias horas después del evento de hipoglucemia) y se preservó la respuesta hormonal contrarreguladora.(43)

Los resultados mostraron que la tasa global de hipoglucemia severa (SH) demostrada durante el Ensayo de Control y Complicaciones de la Diabetes (DCCT) ha disminuido y la tasa relativamente alta de SH asociada con el tratamiento intensivo durante el DCCT se ha disipado durante la epidemiología de las Intervenciones y Complicaciones de la Diabetes (EDIC). La ecualización de las tasas de $\mathrm{SH}$ entre los dos grupos de tratamiento originales es atribuible en gran medida a sus niveles de HbA1c similares durante EDIC. Durante EDIC, el riesgo de $\mathrm{SH}$ permanece fuertemente relacionado con la $\mathrm{HbA1c}$, con un aumento de 13 a 15\% en el riesgo de SH por cada disminución del 10\% en HbA1c.(44)

El estudio concluyó que la frecuencia cardiaca aumenta aproximadamente 20 latidos cuando el nivel de glucemia disminuye en comparación con los días de euglicemia. Los niveles de adrenalina en plasma aumentaron significativamente durante la hipoglucemia en comparación con la euglucemia. Los niveles séricos de potasio disminuyeron de manera similar después de la inyección de insulina durante la hipoglucemia y la euglucemia. (45)
Finalmente, para identificar los cuidados de enfermería para prevenir y controlar los eventos de hipoglucemia en pacientes adultos diagnosticados con diabetes mellitus, los resultados reportados en la literatura se interrelacionaron con los factores y características definitorias y se formularon cuatro Diagnósticos de Enfermería definidos por la North
American Nursing Diagnosis Association (NANDA), con sus respectivas metas de acuerdo con la Nursing Outcomes Classification (NOC), las intervenciones establecidas por la Nursing interventions classification (NIC) y las actividades descritas para cada una de ellas (tabla 2). 
Tabla 2. Plan de atención de enfermería

\begin{tabular}{|c|c|}
\hline Diagnóstico de enfermería & $\begin{array}{l}\text { [00179] Riesgo de nivel de glucemia inestable: vulnerable } \\
\text { a variaciones en la glucosa/azícar de la sangre respecto al } \\
\text { rango normal, que pueden poner en peligro la salud. }\end{array}$ \\
\hline $\begin{array}{l}\text { Factores relacionados y características definitorias } \\
\text { reportados en la literatura }\end{array}$ & $\begin{array}{l}\text { - No adherencia al plan terapéutico de la diabetes }{ }^{26,28} \\
\text { - Manejo insuficiente de la diabetes } \\
\text { - Conocimiento insuficiente sobre los factores modificables } \\
\text { - Control inadecuado de la glucemia } \\
\text { - Manejo ineficaz de la medicación }{ }^{21,26,36,42} \\
\text { - Conocimiento insuficiente sobre el manejo de la diabetes }\end{array}$ \\
\hline Objetivos esperados & $\begin{array}{l}\text { [001779] Nivel de glucemia } \\
\text { [2113] Severidad de la hipoglucemia }\end{array}$ \\
\hline $\begin{array}{l}\text { Intervenciones de enfermería reportados en la } \\
\text { literatura }\end{array}$ & $\begin{array}{l}\text { [2130] Manejo de la hipoglucemia }{ }^{32,33,34,35,36,39} \\
\text { [5602] Enseñanza: proceso de enfermedad }{ }^{30,37,38,49} \\
\text { [5616] Enseñanza: medicamentos prescritos }\end{array}$ \\
\hline Actividades & $\begin{array}{l}\text { - Identificar al paciente con riesgo de hipoglucemia. } \\
\text { - Identificar los signos y síntomas de la hipoglucemia. } \\
\text { - Monitorizar la presencia de signos y síntomas de hipoglucemia. } \\
\text { - Administrar hidratos de carbono simples, si está indicado. } \\
\text { - Contactar con los servicios médicos de urgencia, si es necesario. } \\
\text { - Proporcionar información sobre la conveniencia del autocontrol de la hipoglucemia. } \\
\text { - Enseñar al paciente y a la familia los signos y síntomas, factores de riesgo y } \\
\text { tratamiento de la hipoglucemia. } \\
\text { - Aconsejar al paciente que disponga de hidratos de carbono simples en todo momento. } \\
\text { - Fomentar el autocontrol de la glucemia. }\end{array}$ \\
\hline Diagnóstico de enfermería & $\begin{array}{l}\text { [00078] Gestión ineficaz de la salud: patrón de regulación e integración en la vida } \\
\text { diaria de un régimen terapéutico para el tratamiento de la enfermedad y sus } \\
\text { secuelas que no es adecuado para alcanzar los objetivos de salud específicos. }\end{array}$ \\
\hline $\begin{array}{l}\text { Factores relacionados y características definitorias } \\
\text { reportados en la literatura }\end{array}$ & $\begin{array}{l}\text { - Dificultades con el tratamiento prescrito }{ }^{21,22,42} \\
\text { - En su vida diaria hace elecciones ineficaces para alcanzar objetivos de salud } 22,27 \\
\text { - Déficit de conocimiento del régimen terapéutico }{ }^{26,40} \\
\text { - Complejidad del sistema sanitario }{ }^{31,40} \\
\text { - Apoyo social insuficiente } \\
\text { - Percepción de la gravedad de su condición }{ }^{21,27,29,43} \\
\text { - No incluye el régimen de tratamiento en la vida diaria }{ }^{26,27}\end{array}$ \\
\hline Objetivos esperados & [1619] Autocontrol de la diabetes \\
\hline $\begin{array}{l}\text { Intervenciones de enfermería reportados en la } \\
\text { literatura }\end{array}$ & $\begin{array}{l}\text { 5246] Asesoramiento nutricional| }{ }^{26} \\
\text { [1100] Manejo de la nutrición }{ }^{26,35} \\
\text { [5606] Enseñanza: individual }{ }^{24,30,34,36,37,38,39,40} \\
\text { [2380] Manejo de la medicación }{ }^{21,26,32,33,34,35,36,38,44}\end{array}$ \\
\hline Actividades & $\begin{array}{l}\text { - Establecer metas realistas a corto y largo plazo para el cambio del estado nutricional. } \\
\text { - Comentar los gustos y aversiones alimentarias del paciente. } \\
\text { - Comentar los hábitos de compra de comidas y los límites de presupuesto. } \\
\text { - Determinar la ingesta y los hábitos alimentarios del paciente. } \\
\text { - Facilitar la identificación de las conductas alimentarias que se desean cambiar. } \\
\text { - Determinar el estado nutricional del paciente y su capacidad para satisfacer las } \\
\text { necesidades nutricionales. } \\
\text { - Instruir al paciente sobre las necesidades nutricionales (es decir, comentar las } \\
\text { directrices dietéticas y las pirámides de alimentos) }\end{array}$ \\
\hline
\end{tabular}




\begin{tabular}{|c|c|}
\hline & $\begin{array}{l}\text { - Enseñar al paciente a controlar las calorías y la ingesta dietética (p. ej., diario de } \\
\text { alimentos). } \\
\text { - Comprobar la capacidad del paciente para automedicarse, según corresponda. } \\
\text { - Observar los efectos terapéuticos de la medicación en el paciente. } \\
\text { - Revisar periódicamente con el paciente y/o familia los tipos y dosis de } \\
\text { medicamentos tomados. } \\
\text { - Determinar el conocimiento del paciente sobre la medicación. } \\
\text { - Explicar al paciente y/o a los familiares la acción y los efectos secundarios } \\
\text { esperados de la medicación. } \\
\text { - Enseñar al paciente cuándo debe solicitar atención médica. } \\
\text { - Controlar el cumplimiento del régimen de medicación. } \\
\text { - Determinar las necesidades de enseñanza del paciente. } \\
\text { - Valorar el nivel actual de conocimiento y comprensión de contenidos del paciente. } \\
\text { - Dar tiempo al paciente para que haga preguntas y exprese sus inquietudes. }\end{array}$ \\
\hline Diagnóstico de Enfermería & $\begin{array}{l}\text { [00075] Disposición para mejorar el afrontamiento familiar: patrón de manejo de } \\
\text { las tareas adaptativas por parte de la persona de referencia (familiar, persona } \\
\text { significativa o amigo íntimo) implicada en el cambio en la salud del paciente, que } \\
\text { puede ser reforzado. }\end{array}$ \\
\hline $\begin{array}{l}\text { Factores relacionados y características definitorias } \\
\text { reportados en la literatura }\end{array}$ & - Expresa deseos de mejorar la promoción de la salud²2 \\
\hline Objetivo esperado & $\begin{array}{l}\text { [2013] Equilibrio en el estilo de vida. } \\
\text { [2602] Funcionamiento de la familia. } \\
\text { [2604] Normalización de la familia. } \\
\text { [2608] Resiliencia familiar. }\end{array}$ \\
\hline Intervenciones de Enfermería & $\begin{array}{l}\text { [7100] Estimulación de la integridad familiar. } \\
\text { [7150] Terapia familiar. } \\
\text { [7140] Apoyo a la familia. } \\
\text { [4340] Entrenamiento de la asertividad. } \\
\text { [5230] Mejorar el afrontamiento. } \\
\text { [7110] Fomentar la implicación familiar. }\end{array}$ \\
\hline Actividades & $\begin{array}{l}\text { - Facilitar la armonía dentro de/entre la familia. } \\
\text { - Proporcionar información regularmente a los miembros de la familia sobre el estado } \\
\text { del paciente, de acuerdo con los deseos de éste. } \\
\text { - Colaborar con la familia en la solución de problemas y en la toma de decisiones. } \\
\text { - Escuchar a los miembros de la familia. } \\
\text { - Establecer una relación de confianza con los miembros de la familia. } \\
\text { - Facilitar una comunicación abierta entre los miembros de la familia. } \\
\text { - Establecer los cuidados del paciente por parte de los miembros de la familia, si } \\
\text { resulta adecuado. } \\
\text { - Proporcionar educación e información. } \\
\text { - Compartir el plan de terapia con la familia. } \\
\text { - Asegurar a la familia que al paciente se le brindan los mejores cuidados posibles. } \\
\text { - Responder a todas las preguntas de los miembros de la familia o ayudarles a } \\
\text { obtener las respuestas. } \\
\text { - Incluir a los miembros de la familia junto con el paciente en la toma de decisiones } \\
\text { acerca de los cuidados, cuando resulte apropiado. }\end{array}$ \\
\hline Diagnóstico de Enfermería & $\begin{array}{l}\text { [00126] Conocimientos deficientes: } \\
\text { carencia o deficiencia de información cognitiva relacionada con un tema } \\
\text { específico }\end{array}$ \\
\hline $\begin{array}{l}\text { Factores relacionados y características definitorias } \\
\text { reportados en la literatura }\end{array}$ & $\begin{array}{l}\text { - Comportamientos inapropiados } 22,26,28 \\
\text { - Conocimiento insuficiente } \\
\text { - Información insuficiente }{ }^{40}\end{array}$ \\
\hline
\end{tabular}




\begin{tabular}{|c|c|}
\hline Objetivo esperado & $\begin{array}{l}\text { [1820] Conocimiento: Control de la diabetes } \\
\text { [00075] Disposición para mejorar el afrontamiento familiar: patrón de manejo de } \\
\text { las tareas adaptativas por parte de la persona de referencia (familiar, persona } \\
\text { significativa o amigo íntimo) implicada en el cambio en la salud del paciente, que } \\
\text { puede ser reforzado. }\end{array}$ \\
\hline $\begin{array}{l}\text { Intervenciones de Enfermería reportados } \\
\text { en la literatura }\end{array}$ & $\begin{array}{l}\text { [5520] Facilitar el aprendizaje } 24,30,37,38,40 \\
\text { [5540] Potenciación de la disposición de aprendizaje } e^{30,37,38,40}\end{array}$ \\
\hline Actividades & $\begin{array}{l}\text { - Comenzar la instrucción cuando el paciente demuestre estar preparado para } \\
\text { aprender. } \\
\text { - Establecer metas de aprendizaje realistas con el paciente. } \\
\text { - Identificar claramente los objetivos del aprendizaje y en términos observables. } \\
\text { - Ajustar la instrucción al nivel de conocimientos y comprensión del paciente. } \\
\text { - Adaptar la información para que cumpla con el estilo de vida/rutina del paciente. } \\
\text { - Proporcionar materiales educativos para ilustrar la información importante y/o } \\
\text { compleja. } \\
\text { - Utilizar un lenguaje familiar. } \\
\text { - Explicar la terminología que no sea familiar. } \\
\text { - Fomentar la participación activa del paciente. } \\
\text { - Fomentar que el paciente comparta experiencias válidas durante la experiencia de } \\
\text { aprendizaje. } \\
\text { - Responder a las preguntas de una forma clara y concisa. } \\
\text { - Proporcionar retroalimentación frecuente acerca del progreso del aprendizaje. } \\
\text { - Proporcionar folletos informativos, vídeos y recursos «on line» cuando sea } \\
\text { adecuado. }\end{array}$ \\
\hline
\end{tabular}

Fuente: las autoras, citado de NNN Consult.

\section{IS C USIÓN}

Los estudios permiten evidenciar necesidades explícitas de los pacientes con DM, que presentan eventos de hipoglucemia, a partir de los cuales se identifican diagnósticos, resultados esperados e intervenciones de enfermería. En primer lugar el déficit de educación al paciente con DM, según un estudio realizado la educación referente a la diabetes se considera parte fundamental y es un requisito imprescindible para asumir el control de la enfermedad e integrarla a la vida cotidiana, para esto se debe crear un programa planificado y flexible que cubra las necesidades clínicas individuales teniendo como base la información, motivación, capacitación de habilidades, modificación de hábitos, conductas y conocimiento del uso y gestión del sistema sanitario; para la implementación del programa de educación se deberá valorar el grado de conocimiento, la actitud del paciente y la participación en su tratamiento, así como la situación psicosocial y aspectos clínicos. ${ }^{46}$

La deficiencia de acompañamiento por parte de la familia es importante porque la diabetes no solo afecta al paciente sino también a su familia ya que se producen cambios que involucran a todos los integrantes, como por ejemplo el reajuste de horarios familiares por las diversas rutinas de actividad física del paciente y el cambio en la alimentación. El diagnóstico es relevante porque si no existe red de apoyo familiar el paciente se sentirá solo y se genera mayor estrés. Con el fin de solucionar la situación es necesario asesorar a la familia y explicar la importancia de su apoyo al paciente y las estrategias que pueden seguir para evitar el cansancio del cuidador, si se logra disminuir esta necesidad se obtendrá mayor adherencia al tratamiento, disminución en el nivel de estrés, mejor calidad de vida y bienestar emocional alcanzando la meta de una atención integral. ${ }^{47,48}$

La atención del paciente debe ser individual y tener en cuenta: la clasificación de la diabetes, el entorno psicosocial del paciente y las diferentes comorbilidades de cada uno, con el fin de detectar de manera oportuna las señales que indican la presencia de una complicación, es por esto que el paciente deberá generar una comunicación con su médico para que le sea explicado el tratamiento, ajustándolo a sus diferencias individuales y específicas para un manejo adecuado de la enfermedad, cuando la información no sea suficiente debe ser complementada por el profesional de enfermería. ${ }^{49}$ Con relación al déficit en la capacitación del equipo de salud, una revisión de la literatura resalta la importancia de los conocimientos fisiopatológicos de la DM para la atención de los pacientes. Sin embargo, se evidenció que los profesionales de enfermería priorizan la gestión administrativa sobre el cuidado directo de la salud a las personas en forma individualizada. Por tal motivo es indispensable realizar una actualización enfocada en diabetes, profundizando en temas como la educación 
terapéutica, percepción de la enfermedad, el síndrome metabólico, dieta y ejercicio, tratamiento farmacológico e importancia en la adherencia, evaluación, cuidado psicosocial y complicaciones de la DM. ${ }^{50}$

A partir de lo anterior se realiza una asignación de diferentes diagnósticos de enfermería, objetivos esperados e intervenciones que se interrelacionan a las necesidades descritas por la literatura científica (tabla 2). ${ }^{51}$

\section{CONCLUSIONES}

A partir de las necesidades reportadas en la literatura se determinaron las características definitorias y factores relacionados que permitieron formular cuatro diagnósticos de enfermería orientados a prevenir y controlar los eventos de hipoglucemia en pacientes adultos diagnosticados con DM: riesgo de nivel de glucemia inestable, gestión ineficaz de la salud, disposición para mejorar el afrontamiento familiar y conocimientos deficientes. Así mismo, se determinaron como principales intervenciones de enfermería: enseñanza del proceso de enfermedad, medicamentos prescritos, entrenamiento del asertividad, manejo de la hipoglicemia, nutricional y de la medicación, mejorar el afrontamiento, enseñanza individual, facilitar el aprendizaje y potenciación de la disposición de aprendizaje.

Los resultados aportan a la concepción disciplinar del ser humano en un contexto, dado que las intervenciones de enfermería incluyen aspectos relacionados con los valores, las creencias y las experiencias de la persona con DM.

\section{FI NA N C I AC I Ó N}

Estudio financiado por la Fundación Universitaria de Ciencias de la Salud, a través de la convocatoria interna para el fomento de la investigación.

\section{REFERENCIAS}

1. Organización Panamericana de la Salud, Organización Mundial de Salud [Internet]. Diabetes. Estados Unidos; 2012 [citado Abril 10 de 2019]. [aproximadamente 1 pantalla]. Disponible en: https:// www.paho.org/hq/index.php?option=com_content\&view $=$ articl e\&id=6715:2012-diabetes\&Itemid=39446\&lang=es

2. Organización Mundial de la salud [Internet]. Diabetes. Suiza: Organización Mundial de la salud; 2018 [citado Abril 10 de 2019]. [aproximadamente 2 pantallas]. Disponible en: https://www.who. int/es/news-room/fact-sheets/detail/diabetes

3. Asociación Americana de Diabetes [Internet]. Nueva Guía 2019 sobre Diabetes. Estados Unidos: Asociación Americana de Diabetes; 2019 [citado Julio 14 de 2019]. Disponible en: https:// www.intramed.net/contenidover.asp? contenidoid $=93549$
4. Federación Internacional de Diabetes [Internet]. Atlas de la diabetes. Bélgica: Federación Internacional de Diabetes; 2019 [citado Junio 2 de 2020]. [aproximadamente 1 pantalla]. Disponible en: https://www.diabetesatlas.org/es/sections/demographic-andgeographic-outline.html

5. International Diabetes Federation [Internet]. Diabetes in South and Central America. Belgium: International Diabetes Federation; 2017 [cited April 10 de 2019]. [aproximadamente 2 pantallas]. Available in https://www.idf.org/our-network/regions-members/ south-and-central-america/diabetes-in-saca.html

6. Ministerio de Salud y Protección Social [Internet]. Análisis De Situación De Salud: Colombia. 2018 [citado Abril 10 de 2019]. Disponible en: https:/www.minsalud.gov.co/sites/rid/Lists/ BibliotecaDigital/RIDE/VS/ED/PSP/asis-nacional-2017.pdf

7. Organización Mundial de la Salud [Internet]. Informe Mundial sobre la Diabetes. Suiza: Organización Mundial de la salud; 2016 [citado Abril 15 de 2019]. Disponible en: https://apps.who. int/iris/bitstream/handle/10665/254649/9789243565255-spa. pdf? sequence $=1$

8. Ministerio de Salud y Protección Social [Internet]. Seguimiento a las acciones programadas en el nivel nacional y territorial, para el logro de la Megameta en salud. Colombia; 2017 [citado Abril 15 de 2019]. Disponible en: https://www.minsalud.gov.co/sites/rid/ Lists/BibliotecaDigital/RIDE/VS/PP/ENT/seguimiento-megameta1er-semestre-2017.pdf

9. Ministerio de Salud y Protección Social [Internet]. Minsalud propone implementar estrategia de cuatro por cuatro. Colombia; 2012 [citado Abril 15 de 2019]. [aproximadamente 1 pantalla]. Disponible en: https://www.minsalud.gov.co/Paginas/MinSaludpropone-implementar-estrategia-de-cuatro-por-cuatro.aspx

10. Fundación Para la Diabetes [Intenet]. Hipoglucemia. Madrid; 2017 [citado Mayo 17 de 2019]. [aproximadamente 3 pantallas]. Disponible en: https://www.fundaciondiabetes.org/infantil/188/ hipoglucemia-ninos

11. Mezquita-Raya P, Reyes-García R, Moreno-Perez O, Muñoz-Torres M, Merino Torres J, Gorgojo-Martínez J, et al. Documento de posicionamiento: evaluación de la hipoglucemia en el paciente con diabetes mellitus. Grupo de Trabajo de la Diabetes Mellitus de la Sociedad Española de Endocrinología y Nutrición. Endocrinol Nutr. 2013;60(9): 517.e1-517.e18. doi: 10.1016/j. endonu.2013.04.005

12. UK Hypoglycaemia Study Group. Risk of hypoglycaemia in types 1 and 2 diabetes: effects of treatmentmodalities and theirduration. Diabetologia. 2007;50(6):1140-7. doi: 10.1007/s00125-007-0599-y

13. American Diabetes Association. Standards of Medical Care in Diabetes 2017. Diabetes Care. 2017;40(Suppl.1):S4-S5. doi: $10.2337 / \mathrm{dc} 17-\mathrm{S} 004$

14. Vinocour M. Guias ADA 2017 metas glicemias, DM gestacional, hipoglicemias y vacunación [Internet]. 2017 [Citado el 29 de Julio 2019]. Disponible en: http://portal.medicos.cr/ documents/20183/1486612/Gui\%CC\%81as+ADA+2017.pdf/ d72b2305-36a0-49a4-91f0-2a1924cc92a5 
15. Di LorenziBruzzone RM, Bruno L, Pandolfi M, Javiel G, Goñi M. Hipoglucemia en pacientes diabéticos. Rev. Urug. Med. 2017;2(3):51-60. doi: 10.26445/rmu.2.3.3

16. Menezes KDS, Silveira RCCP, Galvão CM. Revisão integrativa: de método de pesquisa para a incorporação evidências na saúde e na enfermagem. Texto Contexto Enferm. 2008;17(4):758-64.

17. Santillán A. Lectura crítica de la evidencia científica. 2014;XXI(63):15-18.

18. PrimoJ. Niveles de evidencia y grados derecomendación [Internet]. España; 2003 [Citado el 17 de mayo 2019]. Disponible en: https://dianacuesta.files.wordpress. com/2009/07/niveles-de-evidencia-y-grados-de-recomendacion 1.pdf

19. Resolución Numero 8430 de 1993: Por la cual se establecen las normas científicas, técnicas y administrativas para la investigación en salud, (1993).

20. Ley 44 de 1993: por la cual se modifica y adiciona la Ley 23 de 1982 y se modifica la Ley 29 de 1944, Diario Oficial No 40.740 (1993).

21. Wohland T, Holstein JD, Patzer OM, Mende M, Tiemann T, Koch-Tessarek C, et al. New risk and protective factors for severe hypoglycaemia in people with type 1 diabetes. Nutrition, Metabolism and Cardiovascular Diseases. 2017;27(5):407-14. doi: 10.1016/j.numecd.2016.12.006

22. Sonoda N, Morimoto A, Ugi S, Morino K, Sekine O, Nemoto K, et al. Predictors for Mild and Severe Hypoglycemia in Insulin-Treated Japanese Diabetic Patients. PloS One. 2015;10(6):e0130584. doi: 10.1371/journal.pone.0130584

23. Moutairou A, Roussel R, Charbonnel B, Leye A, Detournay B, Mohammedi $\mathrm{K}$, et al. Predicting severe hypoglycaemia with self-monitoring of blood glucose in type 1 diabetes. Diabetes \& Metabolism. 2017;43(4):392-4. doi: 10.1016/j.diabet.2016.11.009

24. Rooijackers HM, Wiegers EC, van der Graaf M, Thijssen DH, Kessels RPC, Tack CJ, et al. A Single Bout of High-Intensity Interval Training Reduces Awareness of Subsequent Hypoglycemia in Patients With Type 1 Diabetes. Diabetes. 2017;66(7):1990. doi: $10.2337 / \mathrm{db} 16-1535$

25. Rosenstock J, Fonseca V, Schinzel S, Dain M-P, Mullins P, Riddle $\mathrm{M}$. Reduced risk of hypoglycemia with once-daily glargine versus twice-daily NPH and number needed to harm with NPH to demonstrate the risk of one additional hypoglycemic event in type 2 diabetes: Evidence from a long-term controlled trial. Journal of Diabetes and its Complications. 2014;28(5):742-9. doi: 10.1016/j. jdiacomp.2014.04.003

26. Almanza O, Chia E, De la Cruz A, Tello T, Ortiz P. Frecuencia de factores asociados a hipoglicemia en el adulto mayor diabético admitido en el servicio de emergencia de un hospital nacional. Rev Med Hered. 2017;28(2):93-100. doi: 10.20453/rmh.v28i2.3109

27. Hatle H, Bjørgaas MR, Rø TB, Olsen SE, Åsvold BO. Fear of hypoglycaemia and its relation to hypoglycaemia awareness and symptom intensity in Type 1 diabetes. Diabetes Research and Clinical Practice. 2018;137:213-20. doi: 10.1016/j. diabres.2018.01.014

28. Martyn-Nemeth P, Quinn L, Penckofer S, Park C, Hofer V, Burke L. Fear of hypoglycemia: Influence on glycemic variability and self-management behavior in young adults with type 1 diabetes. J Diabetes Complications. 2017;31(4):735-741. doi: 10.1016/j. jdiacomp.2016.12.015

29. Martyn P, Schwarz F, Mihailescu D, Nemeth J, Quinn L . Fear of hypoglycemia in adults with type 1 diabetes: impact of therapeutic advances and strategies for prevention - a review. J Diabetes Complications. 2016;30(1):167-77. doi: 10.1016/j. jdiacomp.2015.09.003

30. Halperin I, Mukerji G, Maione M, Segal P, Wolfs M, Goguen J, et al. Adult Patient Perspectives on Care for Type 1 and Type 2 Diabetes Across the Institute of Medicine's 6 Domains of Quality. Can J Diabetes. 2018;42(1):36-43. doi: 10.1016/j.jcjd.2017.03.005

31. Hao S, Zhang N, Folta A, Yuan X,Liu L, Fan Li, et al. Inpatient Glycemic Management in Internal Medicine: An Observational Multicenter Study in Nanjing, China. Curr Med Res Opin. 2017;33(8):1371-1377. doi: 10.1080/03007995.2017.1330256

32. International Hypoglycaemia Study Group. Minimizing Hypoglycemia in Diabetes. Diabetes Care. 2015;38(8):1583-91. doi: 10.2337/dc15-0279

33. Andreassen L, Sandberg S, Kristensen G, Solvik U, Skeide R. Nursing home patients with diabetes: Prevalence, drug treatment and glycemic control. Diabetes Res Clin Pract. 2014;105(1):102-9. doi: 10.1016/j.diabres.2014.04.012

34. Jones G, Khan J, Sainsbury C. Is all hypoglycaemia treated as equal? An observational study of how the type of diabetes and treatment prescribed priorto admission influences quality of treatment of inpatient hypoglycaemia. Acta Diabetol. 2017 Mar;54(3):247-250. doi: 10.1007/s00592-016-0940-3

35. Marelli G, Avanzini F, Iacuitti G, Planca E, Frigerio I, Busi G, et al. Effectiveness of a Nurse-Managed Protocol to Prevent Hypoglycemia in Hospitalized Patients with Diabetes. Journal of Diabetes Research. 2015;2015:173956. doi: $10.1155 / 2015 / 173956$

36. Walfridsson A, Sehlberg M, Gillespie U, Dahlkvist J, Johansson H. Diabetes treatment and hypoglycaemic episodes in elderly patients at nursing homes in Uppsala County. Ups J Med Sci. 2016;121(3): 179-183. doi: 10.1080/03009734.2016.1198441

37. Duncan D, Fitzpatrick D. Improving self-referral for diabetes care following hypoglycaemic emergencies: a feasibility study with linked patient data analysis. BMC Emerg Med. 2016;16:13. doi: 10.1186/s12873-016-0078-1

38. Yu-xue C, Zheng-ren L, Ying Y, En-sheng Y, Xing-hua L. Effect of Recurrent Severe Hypoglycemia on Cognitive Performance in Adult Patients with Diabetes: A Meta-analysis. J Huazhong Univ Sci Technolog Med Sci. 2017;37(5):642-648. doi: 10.1007/s1 1596-017-1784-y

39. Vloemans A, Beers J, Wit M, Cleijne W, Rondags S, GeelhoedDuijvestijn P. et al. Research: Educational and Psychological Aspects Keeping safe. Continuous glucose monitoring (CGM) inpersons with Type 1 diabetes and impaired awareness ofhypoglycaemia: a qualitative study. Diabet Med. 2017;34(10):1470-1476. doi: 10.1111/dme.13429

40. Pacheco A, Sande-Lee S, Cássia R, Batista S, Marques J. Effects of a structured education program on glycemic control in type 
1 diabetes. Arch Endocrinol Metab. 2017 Dec;61(6):534-541. doi: 10.1590/2359-3997000000278

41. Duncan E, Fitzpatrick D,Ikegwuonu T, Evans J, Maxwell M. Role and prevalence of impaired awareness of hypoglycaemia in ambulance service attendances to people who have had a severe hypoglycaemic emergency: a mixed-methods study. BMJ Open. 2018; 8(4): e019522. doi: 10.1136/bmjopen-2017-019522

42. Moheet A, Mangia B, Kumar A, Tesfaye A, Eberly C, BaiC, et al. Naltrexone for treatment of impaired awareness of hypoglycemia in type 1 diabetes: A randomized clinical trial. J Diabetes Complications. 2015;29(8):1277-82. doi: $10.1016 / \mathrm{j}$.jdiacomp.2015.08.004

43. Jennum P, Stender K, Rabøl R, Rye N, Chu P, Madsbad S. The Impact of Nocturnal Hypoglycemia on Sleep in Subjects With Type 2 Diabetes. Diabetes Care. 2015 Nov;38(11):2151-7. doi: 10.2337/dc15-0907

44. Gubitosi-Klug R, Braffett B, White N, Sherwin R, Service J, Lachin JM, et at. Risk of Severe Hypoglycemia in Type 1 Diabetes Over 30 Years of Follow-up in the DCCT/EDIC Study. Diabetes Care. 2017 Aug;40(8):1010-1016. doi: 10.2337/dc16-2723

45. Christensen T, Cichosz S, Tarnow L, Randlov J, Kristensen L, Struijk J, et al. Hypoglycaemia and QT interval prolongation in type 1 diabetes - bridging the gap between clamp studies and spontaneous episodes. J Diabetes Complications. 2014;28(5):723-8. doi: 10.1016/j.jdiacomp.2014.03.007
46. Millán Reyes M, Rioja Vázquez R, Muñoz Arias S. Educación diabetológica y cuidados de enfermería en las personas con diabetes en el ámbito extrahospitalario [Internet]. [Córdoba]: Semes, Diabetes Andalucía, Grupo de Enfermería; 2015 [citado Julio 2019]. Disponible en: http://www.semesandalucia.es/wp-content/uploads/2015/07/ educacion-diabetes-enfermeria.pdf

47. Federación Española de Diabetes. Diabetes y Familia [Internet]. Fedesp.es. 2017 [citado 12 Julio 2019]. Disponible en: https:// fedesp.es/podcast/diabetes-y-familias/

48. Molina M. La importancia de la familia en la diabetes [Internet]. CuidatePlus; 2018 [citado 2019 julio 14]; Disponible en: https://cuidateplus.marca.com/bienestar/2018/11/06/ importancia-familia-diabetes-167963.html.

49. Janssen. Diabetes: cada necesidad requiere una atención personalizada [Internet]. 2016 [citado 12 Julio 2019]. Disponible en: https://www.janssen.com/sites/www janssen_com_peru/files/pdf/Diabetes $\% 20$ Cada $\% 20$ Necesidad \% 20Requiere \%20Una \%20Atenci \%C3\%B3n \% 20 Personalizada.pdf

50. Hernandez Paez R, Aponte Garzón L. Educación en diabetes: un aspecto clave de la formación actual en enfermería. Orinoquia [online]. 2014;18(2):78-92.

51. NNN Consult [recurso electrónico]: NANDA, NOC, NIC. [citado 02 Agosto 2019]. Disponible en:https://catalogo.fucsalud.edu. co:2057/nanda/126 